\title{
Broadly tunable carrier envelope phase stable optical parametric amplifier pumped by a monolithic ytterbium fiber amplifier
}

\author{
A. Fernández, ${ }^{1, *}$ L. Zhu, ${ }^{1}$ A. J. Verhoef, ${ }^{1}$ D. Sidorov-Biryukov, ${ }^{1}$ A. Pugžlys, ${ }^{1}$ A. Baltuška, ${ }^{1}$ K.-H. Liao, ${ }^{2}$ \\ Ch.-H. Liu, ${ }^{2}$ A. Galvanauskas, ${ }^{2}$ S. Kane, ${ }^{3}$ R. Holzwarth, ${ }^{4}$ and F. Ö. Ilday ${ }^{5}$ \\ ${ }^{1}$ Institut für Photonik, Technische Universität Wien, Gusshausstrasse 27-29/387, A-1040 Wien, Austria \\ ${ }^{2}$ Center for Ultrafast Optical Science, University of Michigan, Ann Arbor, Michigan 48109-2099, USA \\ ${ }^{3}$ Department of Mathematics, Bridgewater-Raritan Regional High School, Bridgewater, New Jersey 08807, USA \\ ${ }^{4}$ Menlo Systems GmbH, Am Klopferspitz 19, D-82152 Martinsried, Germany \\ ${ }^{5}$ Department of Physics, Bilkent University, Cankaya, Ankara 06800, Turkey \\ *Corresponding author: almadelc@mail.tuwien.ac.at
}

Received May 27, 2009; revised July 26, 2009; accepted July 31, 2009; posted August 11, 2009 (Doc. ID 111797); published September 10, 2009

In an effort to develop a robust and efficient front end for a chirped-pulse parametric amplification chain, we demonstrate a broadband difference-frequency converter driven by a monolithic femtosecond $\mathrm{Yb}$-doped-fiber amplifier and emitting carrier-envelope-offset-free pulses with the energy of tens of nanojoules tunable in the wavelength range from $1200 \mathrm{~nm}$ to beyond $2 \mu \mathrm{m}$. Next to providing these seed pulses, the system enables direct optical synchronization of $\mathrm{Nd}$ - and Yb-doped pump lasers for subsequent parametric amplification. (C) 2009 Optical Society of America

OCIS codes: $320.7110,190.4970$.

The rapidly evolving technology of optical parametric chirped-pulse amplification (OPCPA) [1] offers unique advantages, such as high broadband gain and efficient energy conversion using narrowband picosecond pump pulses. The perennial problems of OPCPA are pump-seed pulse synchronization and the generation of a frequency-detuned, with respect to the pump, compressible broadband seed pulse for the optical parametric amplifier (OPA). Demonstrated approaches to the synchronization of pump-pulse sources include electronic triggering of a seed laser diode for the generation of a nanosecond $Q$-switched pump pulse [2]; active locking of two master oscillators [3]; nonlinear frequency shifting in a photonic crystal fiber (PCF) [4]; and simultaneous seeding of the pump pulse laser and the OPA from an ultrabroadband Ti:sapphire oscillator [5]. IR OP(CP)A systems with passive carrier envelope phase (CEP) stabilization were realized using a broadband difference-frequency-generation (DFG) seed produced via spectral broadening of the output of fullfledged Ti:sapphire [6,7] and $\mathrm{Yb}$ [8] solid-state chirped pulse amplifiers and via DFG between a frequency-shifted and a direct output of a femtosecond Er-doped fiber amplifier $[9,10]$. In this Letter, we demonstrate a drastically simplified seeding/ synchronization approach based on an environmentally stable microjoule energy 200 fs $100 \mathrm{kHz} \mathrm{Yb-}$ doped fiber amplifier (YDFA) operating at $1040 \mathrm{~nm}$. The YDFA serves both as a potential optical seed (synchronization) source for most $\mathrm{Nd}$ and $\mathrm{Yb}$ amplifiers operating around $1 \mu \mathrm{m}$ and as a driver source for the DFG OPA that produces CEP stable near-IR seed pulses. In the DFG OPA, the frequency-doubled output of the YDFA is used for the generation of a whitelight continuum (WLC) seed in bulk sapphire and for its further parametric amplification.

Generation of temporally compressible white-light in bulk media requires high-fidelity $\leq 200 \mathrm{fs}$ pulses to minimize the impact of delayed (e.g., Raman) nonlinearities [11]. Owing to a large amount of higher-order linear and nonlinear dispersion, such pulses are difficult to obtain from YDFA, prompting the use of solid-state oscillators [12] or highly nonlinear fibers [13] for OPA seeding. A successful white-light seeding of an YDFA-driven OPA was demonstrated in [14], where the fidelity of the $1040 \mathrm{~nm}$ pulse was ensured by the use of free-space stretcher/compressor optics. Recently, fiber coupled signal and pump signal delivery for ultrafast fiber amplifiers has been demonstrated in a system delivering $250 \mathrm{~nJ}, 160 \mathrm{fs}$ pulses [15]. In a bid for a robust turn-key alignment-free design, our YDFA system delivering $>9 \mu \mathrm{J}$ pulses consists of a spliced chain of polarization-maintaining (PM) fibers and pigtailed acousto-optic pulse pickers. This architecture allows us to completely dispense with free-space signal and pump coupling.

The experimental layout, consisting of the YDFA, grism pulse compressor, and a type II collinear CEP stable OPA is presented in Fig. 1. The front end of the system is an all PM monolithic fiber chirped-pulse

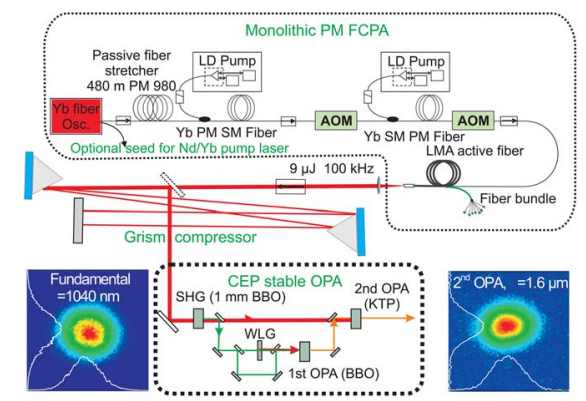

Fig. 1. (Color online) Experimental layout of the MFCPA and DFG/OPA (for details see text). In the lower part farfield mode profiles of the FCPA output (left) and CEP-stable output of DFG/OPA (right) are presented. 
amplifier (MFCPA). It consists of a fiber oscillator, two PM single mode fiber (SMF) preamplifiers, and a PM large mode area (LMA) fiber amplifier. The oscillator delivers $150 \mathrm{fs}$ pulses at a repetition rate of 76 $\mathrm{MHz}$. Before being amplified to $1.6 \mathrm{~nJ}$ at the full repetition rate in the first preamplifier, the pulses are stretched to $\sim 350 \mathrm{ps}$ in $480 \mathrm{~m}$ of a PM SMF (PM980). In the second preamplifier, after reduction of the repetition rate to $100 \mathrm{kHz}$ with an acousto-optic modulator (AOM), the pulses are further amplified to 50 $\mathrm{nJ}$. After the second preamplifier a second AOM is used for suppression of amplified spontaneous emission. In both preamplifiers, $1.5 \mathrm{~m}$ of a highly $\mathrm{Yb}-$ doped PM-SMF from Nufern (PM-YDF-HI) is used as an active medium. Via a mode field adaptor, consisting of a tapered fiber, the preamplified pulses are launched into a fully monolithic LMA stage. This last amplification stage consists of a $3 \mathrm{~m}$ of $\mathrm{Yb}$-doped LMA double-clad fiber and an LMA seed-pump combiner (Nufern PASA-YDF-30/250-7x1). The LMA has a core diameter of $30 \mu \mathrm{m}$, corresponding to a mode field area of $\sim 625 \mu \mathrm{m}^{2}$. After the final amplification stage $>9 \mu \mathrm{J}$ pulses are obtained at a pump power of $\sim 12.5 \mathrm{~W}$ before the active fiber. The far-field beam profile of the MFCPA output is shown in the left inset of Fig. 1.

The pulses from the MFCPA are compressed to sub-200 fs in a negative dispersion compressor based on a pair of grisms made of F2 glass prisms and 1480 lines/mm reflection gratings [16]. The results of the SHG FROG characterization of the compressed pulses are presented in Fig. 2. The measured compressed pulse duration (195 fs) exceeds the calculated transform-limited value of $152 \mathrm{fs}$. The residual chirp originates from a combination of an uncompensated higher-order phase accumulated in the system and a nonlinear phase accumulated in the amplifier $(\sim 6 \pi)$.

Generation of tunable CEP-stable IR seed pulses follows the recipe in [8]. The scheme of our CEP stable OPA is depicted at the bottom of Fig. 1. CEP stable near-IR pulses are produced by generating the difference frequency between the WLC and the second harmonic (SH) of the MFCPA output. The SH (at $520 \mathrm{~nm}$ ) is generated by focusing the entire output of the MFCPA into a 1-mm-thick type I BBO crystal. The frequency doubling efficiency is $46 \%$, owing to the excellent spatial and temporal profile of the compressed MFCPA output. The generated $520 \mathrm{~nm}$ light and remaining fundamental radiation at $1040 \mathrm{~nm}$
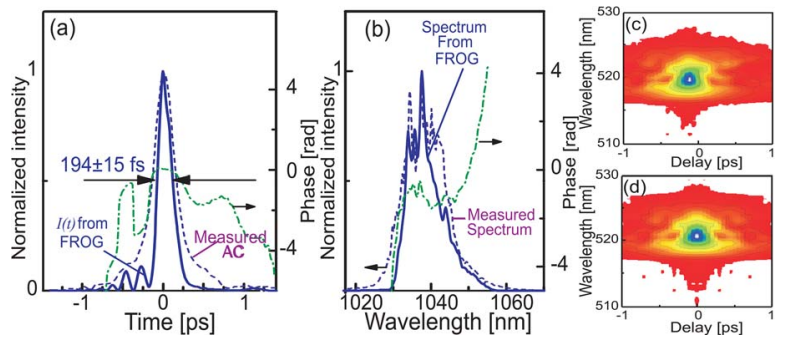

Fig. 2. (Color online) SHG FROG characterization of the MFCPA output. (a) Retrieved temporal pulse profile and temporal phase and measured autocorrelation function. (b) Measured and retrieved spectra together with the spectral phase. (c) Measured and (d) retrieved SHG FROG traces. are separated by a dichroic mirror (DM). The transmitted remaining $1040 \mathrm{~nm}$ light is used for post amplification of the CEP stable IR pulses. A thin-film polarizer in combination with a half-wave plate is used to split the $520 \mathrm{~nm}$ light into two parts: $0.9 \mu \mathrm{J}$ are used to generate a WLC, while the remaining $1.2 \mu \mathrm{J}$ are used as a pump for the first OPA stage. The WLC extends beyond $900 \mathrm{~nm}$ and is generated by focusing the $0.9 \mu \mathrm{J}, 520 \mathrm{~nm}$ pulses into a 5 -mm-thick sapphire plate with a $125 \mathrm{~mm}$ focal length lens and is recollimated afterwards. The pump and seed pulses are combined by a spherical DM $(\mathrm{ROC}=-400 \mathrm{~mm})$, which transmits the WLC seed and focuses the pump pulses into a 4-mm-long type II BBO crystal cut at $28^{\circ}$. A lens $(f=250 \mathrm{~mm})$ placed before the DM focuses the recollimated WLC seed pulses to $100 \mu \mathrm{m}$ in diameter and ensures the optimal spatial overlap of the WLC seed and the pump in the amplifier crystal. Since the WLC seed is temporally chirped, the output of the OPA is spectrally tuned by both adjusting the angular phase-matching of the crystal and optimizing the delay between the pump and seed pulses. The tuning properties of the first OPA stage are summarized in Fig. 3. Pulse energies of up to $80 \mathrm{~nJ}$ for the signal and up to $40 \mathrm{~nJ}$ for the idler waves are achieved. The spectral tunability range of the OPA is mainly determined by the spectral characteristics of the WLC seed. Owing to a limited spectral range of the optical spectrum analyzer used in this work, we were unable to directly record spectra in the wavelength range above $1750 \mathrm{~nm}$. Tunability of the signal in the range of 700-850 $\mathrm{nm}$ is demonstrated in Fig. 3 (a). From the measured tunability of the signal we can infer that the idler wave is tunable from $1200 \mathrm{~nm}$ to beyond $2 \mu \mathrm{m}$.

For further amplification the IR CEP-stable idler beam is focused into a 6 -mm-thick type II KTP crystal $\left(\theta=45.5^{\circ}\right)$ with a $20 \mathrm{~cm}$ focal length lens. The second OPA stage is pumped by the residual $1040 \mathrm{~nm}$ pulse remaining after the $\mathrm{SH}$ generation. The pump beam is focused into the crystal with a $30 \mathrm{~cm}$ focal length lens. The diameter of the seed and the pump beams in the crystal are approximately equal and measure $\sim 120 \mu \mathrm{m}$ at the $1 / e^{2}$ level. For the compen-

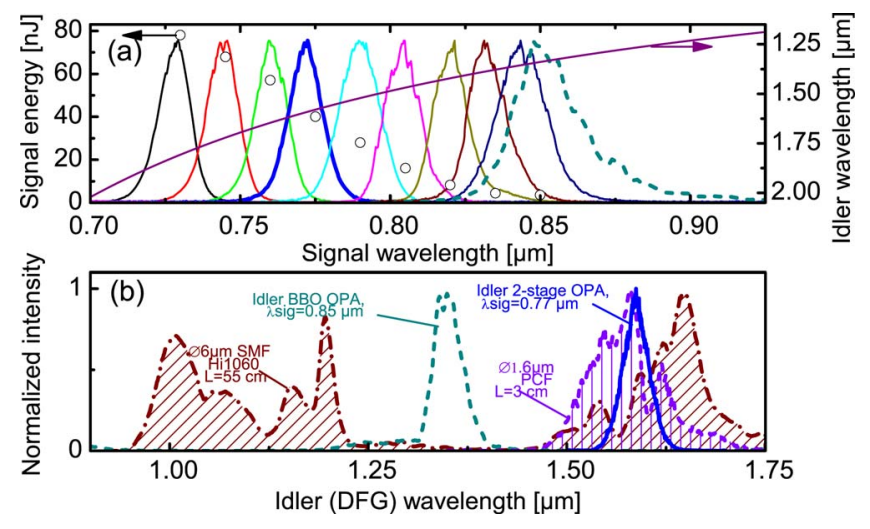

Fig. 3. (Color online) OPA performance. (a) Tunability of first stage OPA. (b) CEP-stable idler spectra at selected wavelengths and results of spectral broadening of the $1.6 \mu \mathrm{m}$ pulse in negative-dispersion PCF (zero dispersion point $750 \mathrm{~nm}$ ) and SMF (Hi1060). 
sation of the walk-off in the KTP crystal the pump and seed beams are focused into the KTP crystal in a slightly noncollinear geometry (noncollinearity angle of $\sim 2^{\circ}$ ). A factor of 4 amplification is achieved in the second OPA stage corresponding to pulse energies of up to $160 \mathrm{~nJ}$. The relative inefficiency of the second stage OPA is explained by a heavy depletion of the fundamental pulse after the frequency doubling stage. The spectrum of the amplified $1.6 \mu \mathrm{m}$ pulses [Fig. 3(b)] has a bandwidth of $43 \mathrm{~nm}$ FWHM, which supports sub-100 fs transform limited pulses.

The CEP stability of the OPA output is confirmed with an $f$-to- $2 f$ interferometer $[8,17]$. Pulses centered at around $1.6 \mu \mathrm{m}$ are spectrally broadened in $55 \mathrm{~cm}$ of Nufern HI-1060 SM fiber [see Fig. 3(b)]. Part of the generated continuum in the spectral range 1820 $1890 \mathrm{~nm}$ is frequency doubled in a $400-\mu \mathrm{m}$-thick BBO crystal and coincided with the short wavelength part of the broadened spectrum (910-945 nm). The temporal overlap is controlled by a delay-line installed in the short-wavelength interferometer arm. The resulting $f$-to- $2 f$ interferograms in the range from 910 to $945 \mathrm{~nm}$ are recorded with an Ocean Optics USB 2000 spectrometer. The observation of stable fringes (Fig. 4) directly proves the CEP stability of the generated IR pulses. Slow phase deviations are caused mainly by mechanical instabilities of the OPA arms and the two arms of the $f$-to- $2 f$ interferometer. In addition beam-pointing instabilities while coupling the $1.6 \mu \mathrm{m}$ light into the SM fiber result in intensity fluctuations, giving rise to an additional source of phase noise $[17,18]$. Recently an all-fiber source delivering stable two-cycle pulses at a center wavelength of $1.17 \mu \mathrm{m}$ was demonstrated via generation of a smooth supercontinuum in a highly nonlinear germano-silica bulk fiber (HNF) [19]. Using a similar approach our OPA source can be used to generate few-cycle pulses in the IR region.

In conclusion, we present a robust directly diodepumped OPCPA front-end that provides straightforward optical synchronization with $\mathrm{Nd} / \mathrm{Yb}$ pump lasers and emits tens of nanojoules of CEP-stable and

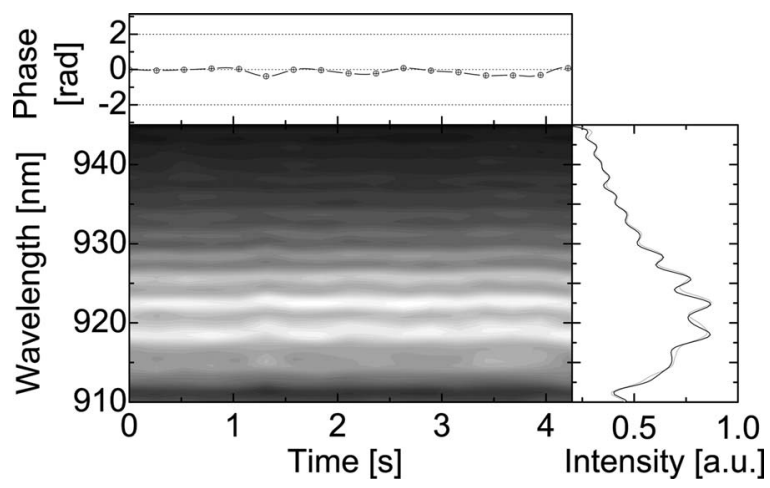

Fig. 4. Measured phase stability with an $f$-to- $2 f$ interferometer after spectrally broadening the OPA output pulses in a SMF. The retrieved phase for each measured spectrum (integrated over 10,000 pulses) is shown in the upper panel. The first (gray curve) and averaged spectrum ( $>4 \mathrm{~s}$ ) (black curve) are shown in the right panel. pulse-pedestal-free DFG light, which is sufficient for overriding the superfluorescence background in multimillijoule OPCPA. To our knowledge this is the first demonstration of a widely tunable CEP-stable submicrojoule OPA pumped by a completely monolithic fiber-based system.

This work has been supported by the Austrian Science Fund (FWF), grants U33-N16 and F1619-N08, and by the Austrian Research Promotion Agency (FFG), grant Eurostars 4319. We are grateful to A. Zheltikov for helpful discussions and to K. Regelskis for technical support. A. F. acknowledges support from a Hertha Firnberg Fellowship by FWF (project T420-N16).

\section{References}

1. A. Dubietis, R. Butkus, and A. P. Piskarskas, IEEE J. Sel. Top. Quantum Electron. 12, 163 (2006).

2. A. Galvanauskas, A. Hariharan, D. Harter, M. A. Arbore, and M. M. Fejer, Opt. Lett. 23, 210 (1998).

3. S. Witte, R. Th. Zinkstok, A. L. Wolf, W. Hogervorst, W. Ubachs, and K. S. E. Eikema, Opt. Express 14, 8168 (2006)

4. C. Y. Teisset, N. Ishii, T. Fuji, T. Metzger, S. Köhler, R. Holzwarth, A. Baltuška, A. M. Zheltikov, and F. Krausz, Opt. Express 13, 6550 (2005).

5. N. Ishii, C. Y. Teisset, T. Fuji, S. Köhler, K. Schmid, L. Veisz, A. Baltuška, and F. Krausz, IEEE J. Sel. Top. Quantum Electron. 12, 173 (2006).

6. C. Vozzi, F. Calegari, E. Benedetti, S. Gasilov, G. Sansone, G. Cerullo, M. Nisoli, S. De Silvestri, and S. Stagira, Opt. Lett. 32, 2957 (2007).

7. X. Gu, G. Marcus, Y. Deng, T. Metzger, C. Teisset, N. Ishii, T. Fuji, A. Baltuška, R. Butkus, V. Pervak, H. Ishizuki, T. Taira, T. Kobayashi, R. Kienberger, and F. Krausz, Opt. Express 17, 62 (2009).

8. O. D. Mücke, D. Sidorov, P. Dombi, A. Pugžlys, A. Baltuška, S. Ališauskas, V. Smilgevičius, J. Pocius, L. Giniunas, R. Danielius, and N. Forget, Opt. Lett. 34, 118 (2009).

9. C. Erny, C. Heese, M. Haag, L. Gallmann, and U. Keller, Opt. Express 17, 1340 (2009).

10. O. Chalus, Ph. K. Bates, M. Smolarski, and J. Biegert, Opt. Express 17, 3587 (2009).

11. G. P. Agrawal, Nonlinear Fiber Optics, 4th ed. (Academic, 2006).

12. J. Rothhardt, S. Hädrich, D. N. Schimpf, J. Limpert, and A. Tünnermann, Opt. Express 15, 16729 (2007).

13. A. Killi, A. Steinmann, G. Palmer, U. Morgner, H. Bartelt, and J. Kobelke, Opt. Lett. 31, 125 (2006).

14. C. Schriever, S. Lochbrunner, P. Krok, and E. Riedle, Opt. Lett. 33, 192 (2008).

15. P. K. Mukhopadhyay, K. Özgören, I. L. Budunoglu, and F. Ö. Ilday, IEEE J. Sel. Top. Quantum Electron. 15, 145 (2009).

16. L. Kuznetsova, F. W. Wise, S. Kane, and J. Squier, Appl. Phys. B 88, 515 (2007).

17. A. Baltuška, M. Uiberacker, E. Goulielmakis, R. Kienberger, V. Yakovlev, T. Udem, T. W. Hänsch, and F. Krausz, IEEE J. Sel. Top. Quantum Electron. 9, 972 (2003).

18. C. Li, E. Moon, H. Wang, H. Mashiko, C. Nakamura, J. Tackett, and Z. Chang, Opt. Lett. 32, 796 (2007).

19. A. Sell, G. Krauss, R. Scheu, R. Huber, and A. Leitenstorfer, Opt. Express 17, 1070 (2009). 